\title{
Optimization of detection strategies for epithermal neutron spectroscopy using photon-sensitive detectors
}

Cite as: Rev. Sci. Instrum. 90, 073901 (2019); https://doi.org/10.1063/1.5091084

Submitted: 01 February 2019 . Accepted: 15 June 2019. Published Online: 09 July 2019

Pierfrancesco Ulpiani (D), Giovanni Romanelli (D), Dalila Onorati (D), Alexandra Parmentier (D), Giulia Festa, Erik Schooneveld, Carlo Cazzaniga, Laura Arcidiacono, Carla Andreani, and Roberto Senesi
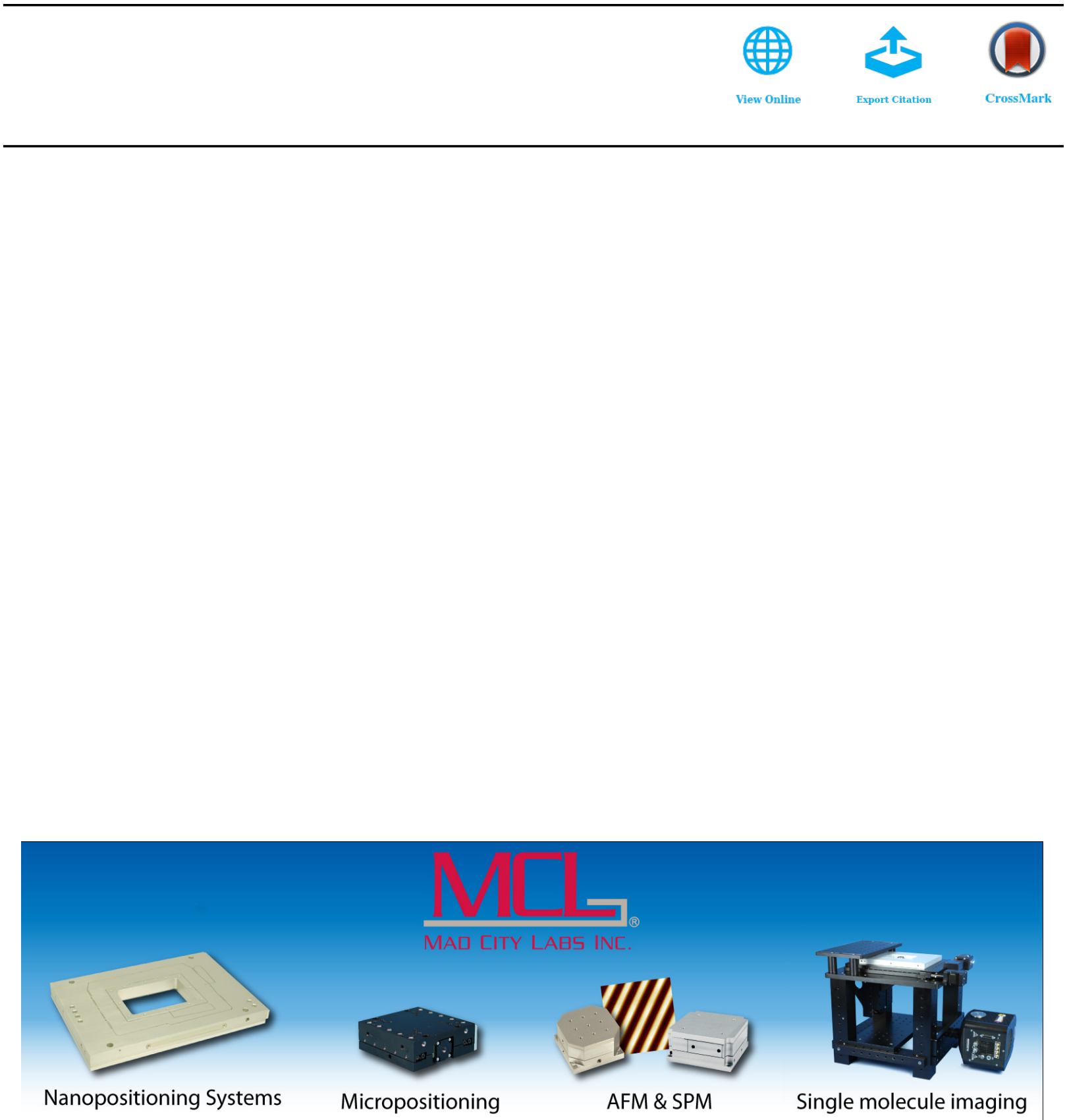


\title{
Optimization of detection strategies for epithermal neutron spectroscopy using photon-sensitive detectors
}

Cite as: Rev. Sci. Instrum. 90, 073901 (2019); doi: 10.1063/1.5091084

Submitted: 1 February 2019 - Accepted: 15 June 2019 •

Published Online: 9 July 2019

\begin{abstract}
Pierfrancesco Ulpiani,, ${ }^{1,2}$ (D) Giovanni Romanelli,, 3 (D) Dalila Onorati, ${ }^{2,4}$ (D) Alexandra Parmentier, ${ }^{5}$
Giulia Festa, ${ }^{6}$ Erik Schooneveld, ${ }^{3}$ Carlo Cazzaniga, ${ }^{3}$ Laura Arcidiacono, ${ }^{2,6,7}$ Carla Andreani, ${ }^{2,4,6,8}$ and Roberto Senesi $i^{2,4,6,8}$
\end{abstract}

\begin{abstract}
AFFILIATIONS
${ }^{1}$ Università degli studi di Roma "Tor Vergata", Dipartimento di Scienze e Tecnologie Chimiche, Via della Ricerca Scientifica 1, Rome, 00133, Italy

Università degli studi di Roma "Tor Vergata", Centro NAST, Via della Ricerca Scientifica 1, Rome, 00133, Italy

${ }^{3}$ ISIS Facility, Rutherford Appleton Laboratory, Chilton, Didcot, Oxfordshire OX11 OQX, United Kingdom

“Università degli studi di Roma "Tor Vergata", Dipartimento di Fisica, Via della Ricerca Scientifica 1, Rome, 00133, Italy

${ }^{5}$ INFN Sezione di Roma Tor Vergata, Via della Ricerca Scientifica 1, I-00133 Rome, Italy

${ }^{6}$ Centro Fermi - Museo Storico della Fisica e Centro Studi e Ricerche "Enrico Fermi", Piazza del Viminale 1, Rome, 00184, Italy

${ }^{7}$ UCL-Institute of Archaeology, University College of London, 31-34 Gordon Square, London WC1H OPY, United Kingdom

${ }^{8} \mathrm{CNR}$-IPCF Sezione di Messina, Viale Ferdinando Stagno d'Alcontres 37, Messina, 98158, Italy
\end{abstract}

a) Electronic mail: giovanni.romanelli@stfc.ac.uk.

\begin{abstract}
In this work, we discuss an improved detection procedure for the photon-sensitive yttrium-aluminum-perovskite detectors installed on the VESUVIO spectrometer at the ISIS pulsed neutron and muon source. By decreasing the low-level energy threshold of detected photons, we observe an increased count rate up to a factor $\sim 3$, and a decrease of relative error bars and noise of $\sim 40 \%$ and $35 \%$, respectively, for deep inelastic neutron scattering measurements. In addition, we demonstrate how the reported optimization may increase the accuracy in the line shape analysis of neutron Compton profiles, as well as in the application of the mean-force approach to detect the anisotropy and anharmonicity in the single-particle local potential. We envisage that such an upgrade of the detection procedure would have a substantial impact on the VESUVIO scientific programme based on deep inelastic neutron scattering investigations.
\end{abstract}

Published under license by AIP Publishing. https://doi.org/10.1063/1.5091084

\section{INTRODUCTION}

Nuclear quantum effects (NQEs), such as zero-point energies, tunneling, and dynamic changes due to isotopic substitution, are attracting increasing attention in the context of aqueous and complex systems, ${ }^{1-7}$ materials in extreme conditions, ${ }^{8}$ and quantum crystals.' Recent studies have shown how hydrogen transfer in enzyme systems is increased by nuclear tunneling ${ }^{10}$ and that NQEs enhance, by orders of magnitude, the exciton diffusion length in organic semiconductors. ${ }^{11}$ Furthermore, the interpretation of anisotropic displacement parameters, both in X-ray and neutron diffraction, relies on the correct modeling of the anisotropy and anharmonicity of the local potentials affecting nuclei, with the resulting necessity to include NQEs in calculations based on both lattice and molecular dynamics. ${ }^{12}$

Deep Inelastic Neutron Scattering (DINS) is an experimental technique providing unique information about NQEs, as it probes the local potential affecting nuclei in materials. ${ }^{1,14}$ For instance, the anisotropic nature of the local potential affecting hydrogen in amorphous ices was experimentally accessed in recent investigations, ${ }^{15}$ and DINS results provided an essential benchmark for state-of-theart path-integral molecular dynamics simulations of light water 
and ice, ${ }^{18}$ and heavy water. ${ }^{19}$ DINS makes use of epithermal neutrons readily available at spallation neutron sources such as the ISIS pulsed neutron and muon source in the UK. ${ }^{20}$ Epithermal neutrons allow for high values of the energy and momentum transfers, thus making it possible to exploit the regime of the Impulse Approximation (IA) ${ }^{21}$ to access the Nuclear Momentum Distributions (NMDs) of atoms in a given sample. VESUVIO ${ }^{22,23}$ at ISIS is the flagship instrument for DINS experiments. VESUVIO is an invertedgeometry spectrometer, ${ }^{24}$ whereby the energy of scattered neutrons is selected at $4.9 \mathrm{eV}$ using a nuclear resonance in ${ }^{197} \mathrm{Au}$, and the Time Of Flight (TOF) technique is used to determine the wave vector and energy transfers for each scattering event. ${ }^{25}$ Photon-sensitive detectors count the prompt radiation following the resonant capture of the neutron, together with the additional background radiation arising from the neutron interaction with blockhouse elements. ${ }^{25-31}$ Figure 1 shows the photon spectrum as recently measured with a high-purity germanium detector on the INES beamline ${ }^{32}$ from a gold sample in correspondence of the resonance at $4.9 \mathrm{eV}$, in red, and outside the resonance, in black. The insert indicates the presence of the main gamma lines from gold after the background correction of the red plot. On VESUVIO, the use of gold foils was preferred to the previous ${ }^{238} \mathrm{U}$ because of safety reasons. Moreover, ${ }^{238} \mathrm{U}$ has less-separated neutron-induced resonances, leading to inconvenient overlap effects on the measured TOF spectra. In the current configuration of the photon-sensitive detectors, a Lower-Level Discrimination Threshold (LLDT) was set at $\sim 600 \mathrm{keV},{ }^{33}$ as pictorially shown in Fig. 1, so as to exclude background photons from boron, present in the instrument blockhouse, (peak A at $\sim 480 \mathrm{keV}$ ), and annihilation (peak B at $511 \mathrm{keV}$ ). ${ }^{34}$ Preliminary results presented in Ref. 35 suggest that the instrument sensitivity can be improved by setting the LLDT to a lower value, so as to detect intense photon peaks in the energy region from $60 \mathrm{keV}$ to $300 \mathrm{keV}$, as evidenced in the insert of the same figure.

Here, we report measurements on polyethylene and gold reference samples on the VESUVIO spectrometer, using the proposed setup on a subset of detectors, showing the improvement of

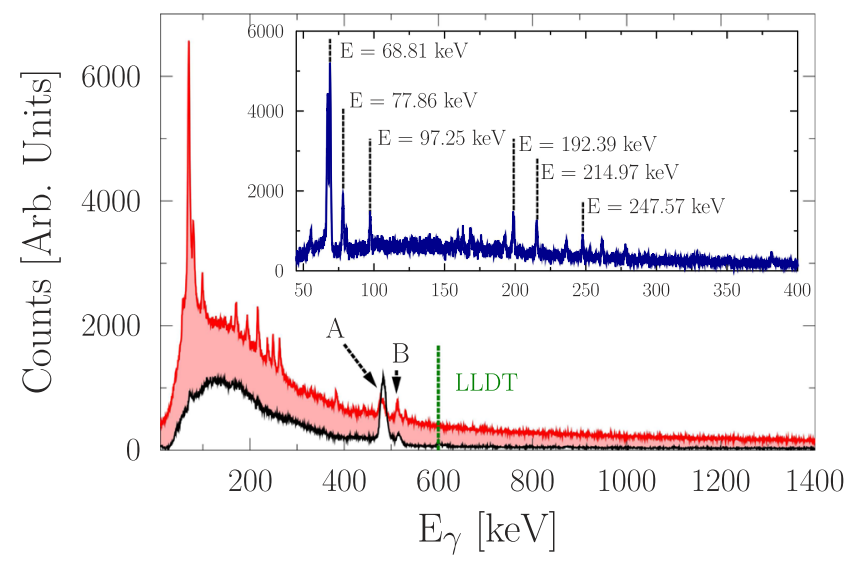

FIG. 1. Photons from gold in correspondence of the resonance at $4.9 \mathrm{eV}$, in red, and outside the resonance, in black, measured on the INES beamline, ${ }^{32}$ using a high-purity germanium detector. The insert indicates the presence of the main gamma lines from gold after the background correction of the red plot. counting statistics and noise reduction on DINS data. Moreover, in Secs. III C and III D, we report estimates on the improvement in the analysis of NMD and Mean Force $(\mathrm{MF})^{16}$ for model systems with anisotropic local potentials, paving the way toward a broader scientific programme of the VESUVIO spectrometer.

\section{MATERIALS AND METHODS}

\section{A. Materials}

DINS measurements were performed on the VESUVIO spectrometer $^{22,23}$ at ISIS on a low-density polyethylene foil and a commercially available Au foil. ${ }^{36}$ The polyethylene sample had a thickness of $250 \mu \mathrm{m}$ along the beam direction, while the Au foil had a thickness of $10 \mu \mathrm{m}$. Both samples were cut so as to have a surface of $\sim 50 \mathrm{~cm}^{2}$ and attached to aluminum frames. The polyethylene foil was inserted in the VESUVIO tank at the sample position, with a vacuum level of $\sim 10^{-5} \mathrm{mbar}$, while the Au foil was placed within the VESUVIO closed-circuit refrigerator, with 60 mbar of He pressure.

\section{B. DINS measurements}

The energy of scattered neutrons was selected by means of a 12.5 - $\mu \mathrm{m}$-thick gold foil; the prompt-gamma radiation was detected by cerium-activated Yttrium-Aluminum-Perovskite (YAP) scintillators; then, the signal was amplified by photomultiplier tubes and discriminated in a Nuclear Instrument Module whenever the deposited energy of the detected photon exceeded the LLDT. Full details of the detection setup are given in Ref. 37. The spectra were recorded using the TOF technique, ${ }^{1}$ whereby the neutron energy transfer is inferred from the time a neutron takes to travel from the source to the detector and, in order to get rid of the large photon background at pulsed neutron sources, ${ }^{38,39}$ the Foil Cycling Technique $(\text { FCT })^{25,40}$ was exploited. In this configuration, an additional gold foil was cycled in and out of the path of scattered neutrons from the sample to the detector. By taking the difference of the two measurements, the sample-independent background was largely suppressed. During the experiment, spectra from 5 forward-scattering detectors, hereafter named from $A$ to $E$, were recorded both with the original LLDT value of $600 \mathrm{keV}$, and with modified values, reported in Table I and discussed in Sec. III B, for a total proton charge in the ISIS synchrotron of $\sim 1500 \mu \mathrm{Ah}$. $^{41}$

\section{Biparametric measurements}

Following the example of Refs. 33 and 42, an additional YAP detector was placed within the VESUVIO blockhouse, at $\sim 90 \mathrm{~cm}$ from the sample position. The scintillator dimensions were $1 \mathrm{in}$. in thickness and 1 in. in diameter. The detector was shielded with a 5 - $\mathrm{cm}$-thick wall of $\mathrm{Pb}$ bricks toward the beam stop, and we noticed that such shielding, not available at present for the other YAP detectors, reduced significantly the background radiation from lowenergy photons. The detector was connected to a negative highvoltage supply of $850 \mathrm{~V}$, and the data were acquired using a pulseshape digitizer CAEN DT5730. This and the above-mentioned YAP detectors were calibrated using ${ }^{137} \mathrm{Cs}$ and ${ }^{60} \mathrm{Co}$ sources ${ }^{33}$ available at ISIS. For each signal from the YAP detector, the digitizer saved the deposited-energy value and a time-stamp value, in the so-called "list mode." By acquiring also the time stamp of 
TABLE I. The setup parameters for the detectors A-E: the value of the LLDT; their position with respect to the sample in polar coordinates $r, \theta$, and $\phi$; the average count rate increase; and the drop of noise and error-bar with respect to the values for the original LLDT.

Detectors LLDT (keV) $\quad r(m) \quad \theta(\mathrm{deg}) \quad \phi(\mathrm{deg}) \quad$ Count-rate increase (\%) $\quad$ Error decrease (\%) Noise (range 1) (\%) Noise (range 2) (\%)

\begin{tabular}{|c|c|c|c|c|c|c|c|c|}
\hline$A$ & $\sim 60$ & 0.51 & 52 & 131 & +200 & -40 & -32 & -35 \\
\hline$B$ & $\sim 170$ & 0.50 & 47 & 140 & +150 & -22 & +4 & -18 \\
\hline C & $\sim 280$ & 0.50 & 44 & 151 & +100 & -12 & -23 & -8 \\
\hline$D$ & $\sim 380$ & 0.51 & 41 & 163 & +20 & -13 & -24 & -6 \\
\hline$E$ & $\sim 490$ & 0.52 & 52 & -132 & +0 & -18 & -20 & -18 \\
\hline
\end{tabular}

the proton-on-target signal of the ISIS accelerator, it was possible to calculate, by difference, the TOF. Using both energy and TOF values, one could populate the biparametric histograms, as in the case of the $10-\mu$ m-thick $\mathrm{Au}$ foil shown in Fig. 2 and
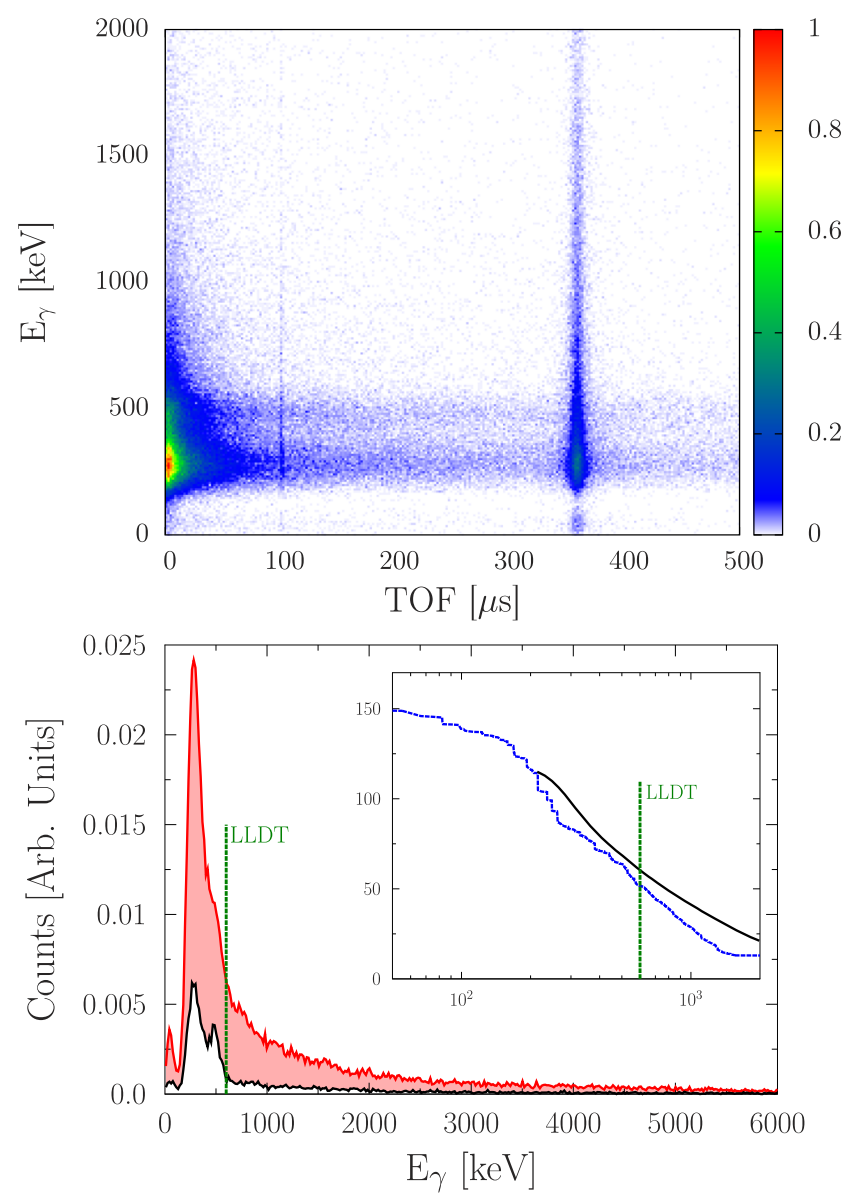

FIG. 2. (Top) Biparametric data from ${ }^{197} \mathrm{Au}$ measured on the VESUVIO spectrometer, as a function of the neutron TOF and photon energy. The color map shows the counts in arbitrary units. (Bottom) The projection on the photon-energy axis of the above data integrated between $340 \mu \mathrm{s}$ and $380 \mu \mathrm{s}$ (red line), and $240 \mu \mathrm{s}$ and $280 \mu \mathrm{s}$ (black line), corresponding to the on- and off-resonance signal from the gold resonance at $4.9 \mathrm{eV}$, respectively. In the insert, the integration of the onresonance signal as a function of the lower limit of integration [keV] (black line), compared to the prediction from Ref. 34 in arbitrary units (blue-dashed line). discussed later. The electronic threshold for the YAP detector used to acquire biparametric spectra was set at 50 channels, corresponding to photon energies of $\sim 200 \mathrm{keV}$ in this particular case.

\section{Neutron Compton profiles}

In DINS experiments, the energy of the incident neutron exceeds the atomic binding energies in the system under investigation, and the process is well described within the framework of the IA. Here, the neutron-scattering intensity is maximized for the values of the energy transfer $E=E_{r}$, with $E_{r}=\hbar^{2} Q^{2} / 2 M$ as the recoil energy, $\hbar Q$ the momentum transfer, and $M$ the mass of the struck nucleus. Because of the dependence upon $M$ of the recoil energy, DINS spectra are composed of a collection of mass-resolved recoil peaks, each of them associated with a neutron Compton profile (NCP) that contains the information about the atomic dynamics. The NCP, $J(y, \hat{\mathbf{Q}})$, is related to the atomic NMD, $\mathrm{n}(\mathbf{p})$, by the relation

$$
J(y, \hat{\mathbf{Q}})=\hbar \int n(\mathbf{p}) \delta(\hbar y-\mathbf{p} \cdot \hat{\mathbf{Q}}) d \mathbf{p},
$$

where $y$ is the West-scaling variable $\hbar y=\mathbf{p} \cdot \hat{\mathbf{Q}},{ }^{43}$ and $\mathbf{p}$ is the momentum vector of the nucleus before the scattering event. The width of the NMD is directly related to the nuclear mean kinetic energy, $\left\langle E_{k}\right\rangle$, and its shape is determined by the curvature, anisotropy, and anharmonicity of the local potential affecting a given nucleus. In the case of a harmonic and anisotropic potential, one can model the NMD using a multivariate Gaussian function of the form

$$
n(p)=\left\langle\Pi_{i} \frac{\hbar}{\sqrt{4 \pi M\left\langle E_{k}\right\rangle_{i}}} \exp \left[-\frac{\hbar^{2} p_{i}^{2}}{4 M\left\langle E_{k}\right\rangle_{i}}\right]\right\rangle,
$$

where $i=x, y, z$ runs over the molecular axes, and a spherical average $\langle\ldots\rangle$ is required for amorphous or polycrystalline samples, where the information about $\mathbf{Q}$ is lost. The mean kinetic energy, in this case, can be expressed as a sum of its directional contributions, $\left\langle E_{k}\right\rangle=\sum_{i}\left\langle E_{k}\right\rangle_{i}$

The analysis of DINS data is carried out with a simultaneous fit for each individual $i$ th detector of the experimental NCP, $F_{i}(y, Q)$, where the asymptotic IA profile of Eq. (1), valid only for infinite values of $Q$, includes corrections due to the finite value of $Q$, $\Delta J_{i}(y, Q)$, known as Final State Effects (FSEs), and it is broadened by the instrument resolution function $\left.R_{i}(y, Q)\right)^{44,45}$

$$
F_{i}(y, Q)=\left[J(y)+\Delta J_{i}(y, Q)\right] \otimes R_{i}(y, Q),
$$


with $R_{i}(y, Q)$ determined using standard Monte Carlo routines available on VESUVIO. ${ }^{46}$ Finally, the experimental NCP is usually represented using the function $\bar{F}(y)$, corresponding to the average over all detectors.

\section{RESULTS}

\section{A. Biparametric spectra}

Figure 2 (top) shows the biparametric spectrum from the $\mathrm{Au}$ sample in the TOF range typical of DINS measurements on VESU$\mathrm{VIO}$ and for photon energies below $2 \mathrm{MeV}$. Vertical lines correspond to neutron-induced resonances at $4.9 \mathrm{eV}$ and $61 \mathrm{eV}$. The horizontal line at $\sim 480 \mathrm{keV}$ represents the main component to the gamma background from the prompt photons emitted by boron in several instrument components. At lower photon energies, the detector efficiency and the background increase, corresponding to a steep increase in the count rate, with an apparent maximum at the detection threshold of $200 \mathrm{keV}$ chosen for this particular case. In Fig. 2 (bottom), the black line corresponds to the biparametric spectrum projected onto the photon-energy axis by integration between $240 \mu \mathrm{s}$ and $280 \mu \mathrm{s}$ (off resonance), and one can recognize the boron peak at $\sim 480 \mathrm{keV}$. In the same figure, the red line is the result of a similar procedure as before with the TOF integration between $340 \mu$ s and $380 \mu$ s, i.e., corresponding to the prompt cascade from the resonance at $4.9 \mathrm{eV}$ (on resonance). One can notice a huge increase between $200 \mathrm{keV}$ and $300 \mathrm{keV}$ in the signal with respect to the off-resonance background. This on-resonance increase is related to the many peaks in the photon emission spectrum of Au already showed in the insert of Fig. 1. One can notice that YAPs, as opposed to the high-purity Ge detector, have lower energy resolution and one cannot distinguish between neighboring photon peaks. On VESU$\mathrm{VIO}$, the DINS signal is proportional to the integral of the onresonance spectrum integrated between the detectable upper limit (around $6 \mathrm{MeV}$ for gold ${ }^{34}$ ), and the lower limit at the value of the LLDT. The blue dashed line in the insert of Fig. 2 (bottom) corresponds to such a numerical integration of the tabulated Au resonance from Ref. 34, and it is compared to the numerical integration of our on-resonance experimental signal (black line) down to the set threshold at $\sim 200 \mathrm{keV}$. The shape of the steplike prediction curve compares very favorably with the experimental one, considering that the latter (i) was obtained with a low-resolution YAP detector not enabling the discrimination of neighbor peaks, and (ii) the measured intensity is partially redistributed between photon peaks and Compton edges. Both curves suggest that the measured signal from $\mathrm{Au}$ can increase up to 2-3 times when the LLDT is lowered between $60 \mathrm{keV}$ and $200 \mathrm{keV}$, as discussed later. One should notice that the LLDT used at present was established when DINS spectra on VESUVIO were collected using a ${ }^{238} \mathrm{U}$ foil. Uranium has less intense photon peaks below $600 \mathrm{keV}$, and they are mainly centered around the background peaks from boron and annihilation, thus explaining the decision to set the LLDT at $600 \mathrm{keV}$ in the first place.

\section{B. Counting error and noise reduction}

Figure 3 shows an example of TOF spectra for detector $A$ with scattering angle $\theta \simeq 52^{\circ}$, in the case of the original LLDT (green squares), and for the lowest value of the LLDT (red circles) used, $E_{0} \sim 60 \mathrm{keV}$. In the top figure, both the original and changed
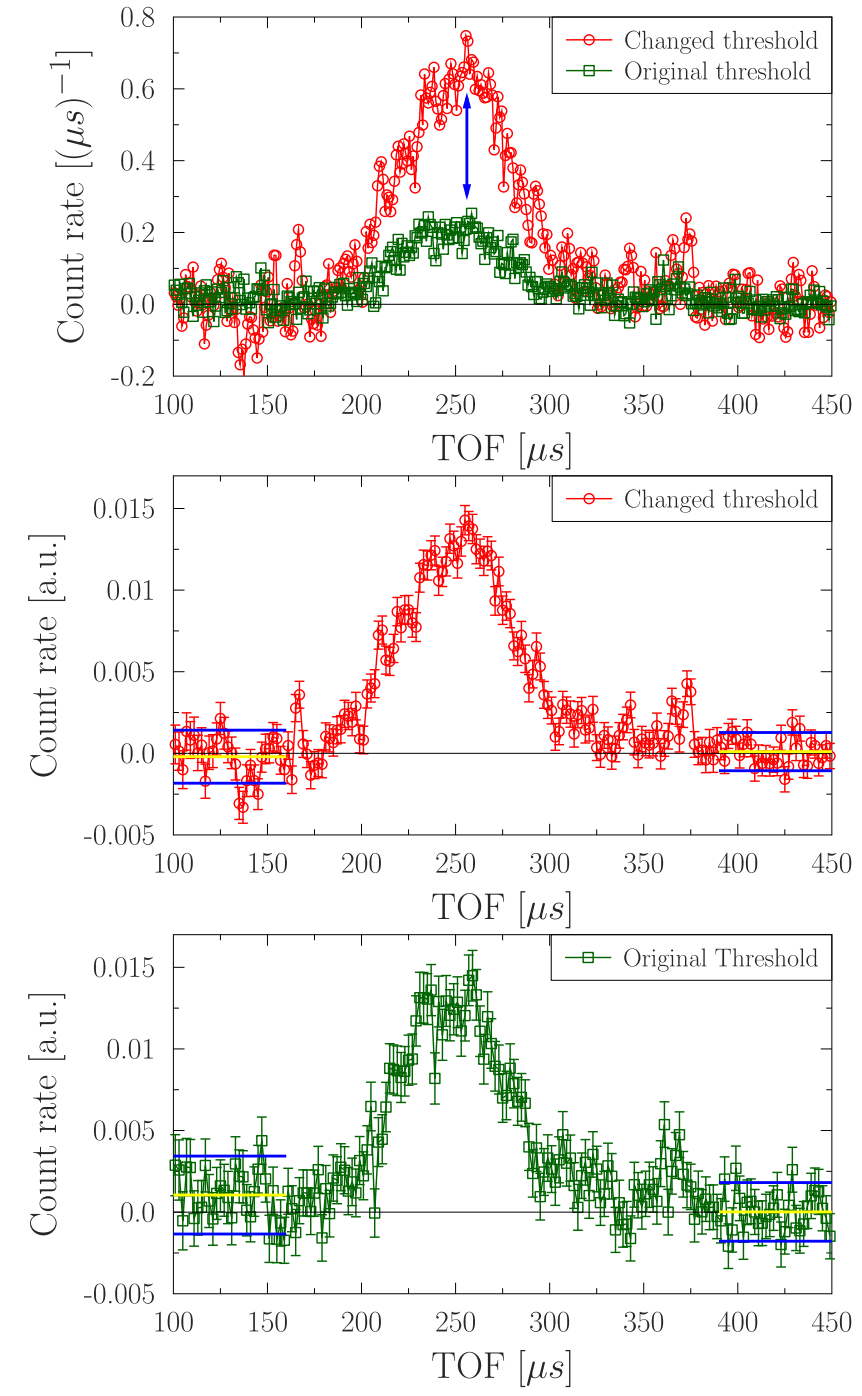

FIG. 3. Spectra from detector $A$ for a polyethylene sample, hydrogen, and carbon DINS peaks are evident at lower and higher TOF, respectively. In the top figure, spectra in both the configurations normalized to the proton charge accumulated in the synchrotron; the blue arrow indicates the increase in the counting statistics of a factor $\sim 3$. Unit-area normalized spectra from detector A recorded with the original (bottom) and modified (middle) LLDT; here, the TOF ranges 1 and 2, where the noise levels have been evaluated, are indicated as blue lines, and the average count rate in the range as yellow line.

configurations are normalized to the proton charge accumulated in the synchrotron, so as to show the increase in the count rate of a factor $\sim 3$. The green spectrum in the bottom and the red one in the middle panel are, instead, unit-area normalized so as to stress the reduction of $\sim 40 \%$ of the error bars when the LLDT is lowered, ${ }^{35}$ and prompt-gamma rays from the gold resonance in the range between $E_{0}$ and $600 \mathrm{keV}$ are also included. Smaller count-rate increases were observed for the other detectors with modified LLDTs, as reported in Table I. In order to quantify the reduction of the noise level in DINS spectra, two TOF ranges were studied where the DINS signal 
is expected to be vanishing. The ranges chosen are to the right of the carbon peak, range $1(390 \mu \mathrm{s}<\mathrm{TOF}<450 \mu \mathrm{s})$, and to the left of the hydrogen peak, range $2(100 \mu \mathrm{s}<\mathrm{TOF}<160 \mu \mathrm{s})$.

The standard deviation, $\Delta c$, of the distribution of counts in these ranges was calculated for the original and changed LLDTs, and it was used to give a measure of the noise and scatter of the data. The noise in the TOF spectra is related to the difference of two count rates in the foil-in and foil-out configurations, ${ }^{25}$ thus being sensitive to the LLDT value. The standard deviation $\Delta c$ is calculated using

$$
\Delta c^{2}=\frac{1}{N-1} \sum_{i}^{N}\left(c\left(t_{i}\right)-\bar{c}\right)^{2},
$$

where $i$ runs over the $N$ bins in the TOF range $\left\{t_{i}\right\}, c\left(t_{i}\right)$ is the count rate in the time interval between $t_{i}$ and $t_{i+1}$, and $\bar{c}$ is the average count rate over the range considered. The values of $\bar{c} \pm \Delta c$ are shown in Fig. 3 for detector $A$ for both TOF ranges considered.

Detector $A$ shows the largest noise reduction of about $35 \%$ on both TOF ranges. On the other hand, detectors $B-D$ show different reductions on the two ranges as listed in Table I. Such behavior can be linked, in first approximation, to the photons emitted by ${ }^{113} \mathrm{Cd}$ in the LLDT energy range investigated, of which the most intense is at $558 \mathrm{keV} .{ }^{34}$ Indeed, at present, the forward-scattering VESUVIO detectors are equipped with 1-mm-thick cadmium foils for calibration purposes. In addition to the $E^{-1 / 2}$-dependent absorption cross section, ${ }^{113} \mathrm{Cd}$ has neutron-induced capture resonances in the neutron-energy range between 10 and $70 \mathrm{eV}$, which roughly corresponds to the TOF values in range 1 for scattered epithermal neutrons. These photons provide counts from the background only, and the present study suggests that the calibration-related cadmium foils should be generally removed during experiments and for any value of the LLDT.

\section{Line shape analysis with optimized setup}

The reduction of noise level and error bars are crucial factors in the analysis of NCPs. Indeed, a more precise line shape analysis of Compton profiles allows for a more detailed determination of the local potential affecting nuclei in a given system. The extent of the benefits from an optimization of the LLDT is discussed below, taking as an example the case of hydrogen in a molecular system with local potential and NMD similar to that of the condensed phases of water. To this aim, we have calculated two hydrogen experimental NCPs for isotropic and anisotropic harmonic potentials, $\bar{F}(y)$, shown in Fig. 4, both of which would result in the same mean kinetic energy. The two $\bar{F}(y)$ functions are calculated using Eq. (3), considering $J(y)$ as the spherically averaged NCP. In particular, the isotropic function is characterized by the mean kinetic energy $\left\langle E_{k}\right\rangle=143.1 \mathrm{meV}$, while the multivariate function corresponds to $\left\langle E_{k}\right\rangle_{x}=8.3 \mathrm{meV},\left\langle E_{k}\right\rangle_{y}=33.2 \mathrm{meV}$, and $\left\langle E_{k}\right\rangle_{z}=101.6 \mathrm{meV}$. Both isotropic and anisotropic NCPs are reported in Fig. 4, together with their difference. The aim of this calculation is to relate the optimization of the LLDT values to an improved experimental sensitivity able to distinguish two NCP line shapes with the same variance, i.e., the same second moment, but with different fourth and sixth moments. 6,47

The anisotropic profile in Fig. 4 is transformed into simulated experimental data by adding typical experimental error bars from VESUVIO measurements, ${ }^{4,48}$ and numerically generated random

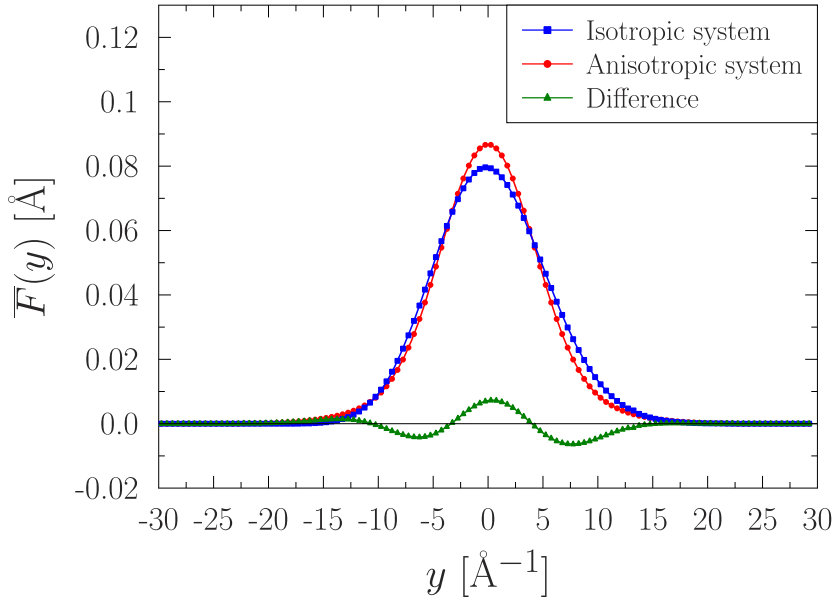

FIG. 4. Calculated isotropic (blue) and anisotropic (red) NCPs as defined in the text, together with their difference (green).

noise. The simulated data are reported in Figs. 5(a) and 5(b). The difference between the calculated isotropic and anisotropic systems (Fig. 4) is also shown for comparison. The right panels of Fig. 5 show enlarged views of the NCP differences between the simulated anisotropic data and the calculated isotropic model. The error bars and noise in Fig. 5(a) are representative of a VESUVIO measurement from a $10 \%$ scattering-power sample measured for $\sim 3600 \mu \mathrm{Ah}$ of the integrated ISIS proton current and acquired with the original LLDT at $600 \mathrm{keV}$. For the same sample, Fig. 5(b) corresponds to the same acquisition time $(3600 \mu \mathrm{Ah})$, but with the error bars and noise as if all detectors had the same modified LLDT as detector A. In particular, the error bars and noise of Fig. 5(a) are reduced by $40 \%$ and 35\%, respectively, as indicated by the results shown in Table I. The random noise is generated using a ROOT script that exploits the TRandom 3 function that produces a random number in a given range. ${ }^{49}$

The single-detector simulated experimental spectra, whose detector average is reported in Fig. 5 in red, were thus fitted using a global fitting procedure using a multivariate Gaussian profile to model the anisotropic momentum distribution as described in Ref. 3. The results are compared to the input parameters for $\left\langle E_{k}\right\rangle_{i}$ in the calculated anisotropic profile and are reported for the two cases in Table II. Results from Fig. 5(a) (case 1) are consistent with the input values, and the associated error bars are of the same order as those reported in recent investigations. ${ }^{4,48}$ The fitted parameters from Fig. 5(b) (case 2), corresponding to the configuration proposed in the present work, are associated with error bars about half of those in the previous case. Such a level of accuracy in the determination of the directional contributions to the mean kinetic energy would represent a valuable benchmark, for example, for computer simulations tackling competing quantum effects from subtle changes in the hydrogen-bonding network in water and ice. ${ }^{3,17}$

\section{Mean-force analysis with optimized setup}

Recently, the MF approach has been used to analyze DINS data $^{16,18,50}$ as it provides a model-independent and nonparametric 

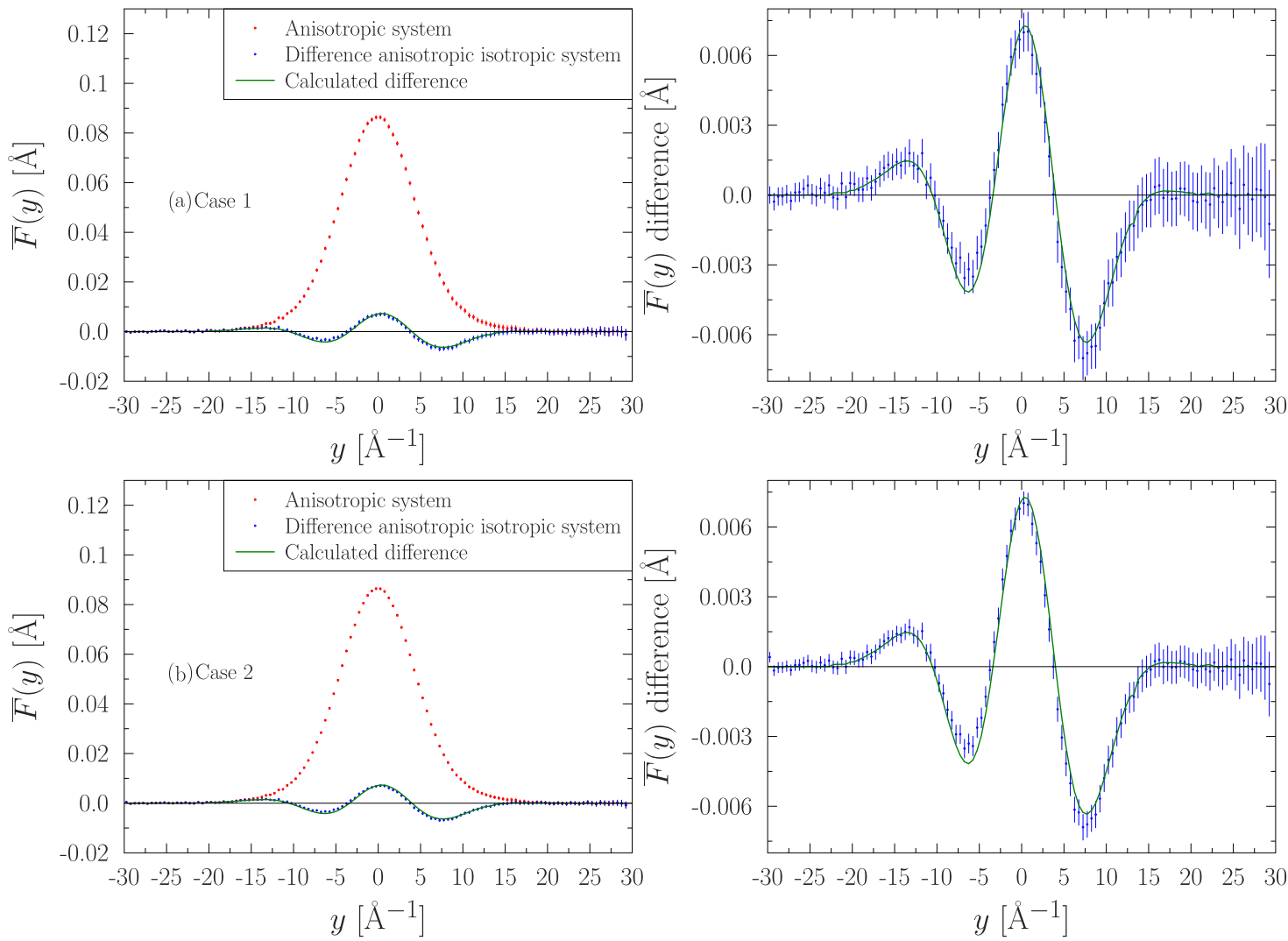

FIG. 5. (a) and (b) Simulated experimental anisotropic $\bar{F}(y)$ profile with error bars and noise in red, the difference with the calculated isotropic $\bar{F}(y)$ in blue, and the difference reported in Fig. 4 in green. On the right of each profile, an enlarged view of the Compton profile differences between the simulated anisotropic data and the calculated isotropic model is shown. Case 1 is representative of a VESUVIO measurement from a $10 \%$ scattering-power sample measured for $\sim 3600 \mu$ Ah of the integrated ISIS proton current and acquired with the original LLDT at $600 \mathrm{keV}$, while case 2 corresponds to the same acquisition time but with the error bars and noise as if all detectors had the same modified LLDT as detector $A$.

tool to highlight differences in systems with various degrees of anharmonicity and anisotropy. The experimental MF function is defined for amorphous or polycrystalline samples as

$$
f\left(x_{\|}\right)=-\frac{M x_{\|}}{\beta^{2} \hbar^{2}}+\frac{1}{\beta} \frac{\int_{0}^{\infty} y \sin \left(x_{\|} y\right) \bar{F}(y) d y}{\int_{0}^{\infty} \cos \left(x_{\|} y\right) \bar{F}(y) d y},
$$

where $x_{\|}$is the projection of the spatial coordinate (displacement) along the direction of the momentum transfer, $\beta=1 / k_{B} T, T$ is the thermodynamic temperature, and $k_{B}$ the Boltzmann's constant. ${ }^{16}$

TABLE II: $\left\langle E_{k}\right\rangle_{i}$ values of the calculated anisotropic $\bar{F}(y)$ and of the simulated experimental $\bar{F}(y)$ in the two cases obtained from the fitting procedure.

\begin{tabular}{lcrr}
\hline \hline$\left\langle E_{k}\right\rangle_{i}$ & Input parameters $(\mathrm{meV})$ & Case 1 $(\mathrm{meV})$ & Case 2 $(\mathrm{meV})$ \\
\hline$\left\langle E_{k}\right\rangle_{x}$ & 8.3 & $7.9 \pm 2.4$ & $8.1 \pm 1.5$ \\
$\left\langle E_{k}\right\rangle_{y}$ & 33.2 & $35.7 \pm 4.3$ & $34.7 \pm 2.7$ \\
$\left\langle E_{k}\right\rangle_{z}$ & 101.6 & $98.4 \pm 4.9$ & $99.7 \pm 2.9$ \\
$\left\langle E_{k}\right\rangle_{t o t}$ & 143.1 & $142.0 \pm 6.9$ & $142.5 \pm 4.2$ \\
\hline \hline
\end{tabular}

The MF is an extension of the Hooke's law applied to atomic displacements in the subnanometer scale, representing the force exerted on an atom in a molecular system, as a function of the displacement from the equilibrium position, and averaged along the Cartesian directions. In fact, a linear relation between the force and the displacement holds for harmonic and isotropic potentials for which one can write $f\left(x_{\|}\right)=\left(-\frac{1}{\beta}+\frac{2\left\langle E_{k}\right\rangle}{3}\right) \frac{M}{\beta \hbar^{2}} x_{\|}$. The DINS technique allows an experimental determination of the mean force and, in the present case, deviations from linearity between displacement and force provide quantitative information on the anisotropy of the effective local potential exerted on hydrogen atoms.

In particular, the MF determined from the simulated experimental NCPs in Figs. 5(a) and 5(b) after the correction of the FSEs [see Eq. (3)] are shown in Figs. 6(a) and 6(b), together with the MF from the isotropic and harmonic system reported in blue in Fig. 4. The anisotropic behavior, i.e., the deviation from linearity, is clearly identified for case 1, where noise and error bars are comparable to the present performance of VESUVIO, for $x_{\|}>0.18 \AA$, while in case 2, the optimized configuration proposed in this work, the discrimination is already possible for $x_{\|}>0.14 \AA$. 

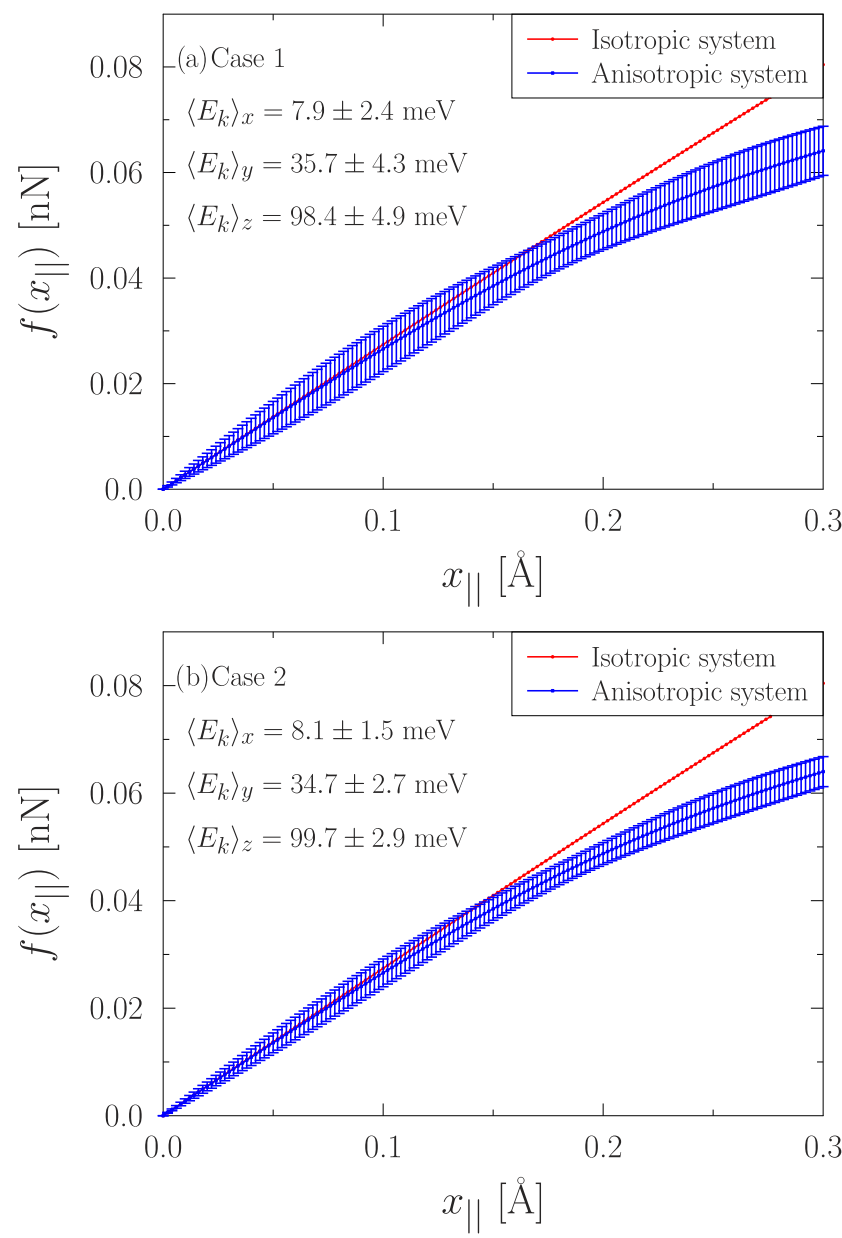

FIG. 6. (a) and (b) MF for calculated isotropic and harmonic system in red and MF for simulated experimental NCPs of Figs. $5(\mathrm{a})$ and $5(\mathrm{~b})$ in blue. The results from the fitting procedure are also reported for the two cases.

\section{OUTLOOK AND CONCLUSIONS}

We have shown how the change of the low-level discrimination thresholds of the gamma-sensitive forward-scattering detectors on the VESUVIO spectrometer allows a substantial increase of the count rates, and a reduction of the experimental error bars and noise levels. In particular, by moving the LLDT value from $600 \mathrm{keV}$ to its minimum value, we have observed a threefold increase in the count rate, and a reduction of $\sim 40 \%$ and $35 \%$ of error bars and noise, respectively. We also noticed that $\mathrm{a} \mathrm{Pb}$ shielding between the YAP detectors and the beam stop can reduce significantly the background radiation from low-energy photons, thus providing a further increase in the signal-to-background ratio. The impact of such improvement on the VESUVIO capabilities, if all LLDT were to be modified in the same way, would allow a line shape analysis on low-scattering power samples, or enhanced parametric studies, e.g., temperature or pressure scans. Future studies will be devoted to the development of window discrimination, or upper-level threshold configurations, which may allow further optimization of the setup.
In addition, the optimized thickness of the analyzer foils in the FCT configuration is under investigation, so as to provide an increased intensity of the analyzer transfer function, paving the way for an increase of approximately an order of magnitude of the global count rate, which would allow enhanced nonparametric line shape analysis of DINS data on VESUVIO.

\section{ACKNOWLEDGMENTS}

This work was partially supported within the CNR-STFC Agreement (2014-2020) concerning collaboration in scientific research at the ISIS pulsed neutron and muon source. The authors gratefully acknowledge the UK Science \& Technology Facilities Council for access to beam time at the ISIS Facility. We also thank Dr. N. Rhodes and A. Spaziani for their help in the setup of the biparametric measurements.

\section{REFERENCES}

${ }^{1}$ C. Andreani, M. Krzystyniak, G. Romanelli, R. Senesi, and F. Fernandez-Alonso, "Electron-volt neutron spectroscopy: Beyond fundamental systems," Adv. Phys. 66(1), 1-73 (2017).

${ }^{2}$ C. Andreani, R. Senesi, M. Krzystyniak, G. Romanelli, and F. Fernandez-Alonso, "Experimental studies of nuclear quantum effects in condensed matter: The case of water," Riv. Nuovo Cimento 41(5), 291-340 (2018).

${ }^{3}$ C. Andreani, G. Romanelli, and R. Senesi, "Direct measurements of quantum kinetic energy tensor in stable and metastable water near the triple point: An experimental benchmark," J. Phys. Chem. Lett. 7(12), 2216-2220 (2016).

${ }^{4} \mathrm{G}$. Romanelli, F. Fernandez-Alonso, and C. Andreani, "The harmonic picture of nuclear mean kinetic energies in heavy water," J. Phys.: Conf. Ser. 571, 012003 (2014).

${ }^{5}$ C. Andreani, D. Colognesi, A. Pietropaolo, and R. Senesi, "Ground state proton dynamics in stable phases of water," Chem. Phys. Lett. 518, 1-6 (2011).

${ }^{6}$ C. Andreani, D. Colognesi, J. Mayers, G. Reiter, and R. Senesi, "Measurement of momentum distribution of lightatoms and molecules in condensed matter systems using inelastic neutron scattering," Adv. Phys. 54(5), 377-469 (2005).

${ }^{7}$ M. Ceriotti, W. Fang, P. G. Kusalik, R. H. McKenzie, A. Michaelides, M. A. Morales, and T. E. Markland, "Nuclear quantum effects in water and aqueous systems: Experiment, theory, and current challenges," Chem. Rev. 116(13), 7529-7550 (2016).

${ }^{8}$ T. E. Markland and M. Ceriotti, "Nuclear quantum effects enter the mainstream," Nat. Rev. Chem. 2, 0109 (2018).

${ }^{9} \mathrm{C}$. Cazorla and J. Boronat, "Simulation and understanding of atomic and molecular quantum crystals,” Rev. Mod. Phys. 89(3), 035003 (2017).

${ }^{10}$ M. J. Sutcliffe and N. S. Scrutton, "A new conceptual framework for enzyme catalysis: Hydrogen tunneling coupled to enzyme dynamics in flavoprotein and quinoprotein enzymes,” Eur. J. Biochem. 269(13), 3096-3102 (2002).

${ }^{11}$ Y. Jiang, Z. Shuai, and M. Liu, "Roles of long-range hopping, quantum nuclear effect, and exciton delocalization in exciton transport in organic semiconductors: A multiscale study," J. Phys. Chem. C 122(32), 18365-18375 (2018).

${ }^{12}$ A. M. Reilly, D. A. Wann, M. J. Gutmann, M. Jura, C. A. Morrison, and D. W. Rankin, "Predicting anisotropic displacement parameters using molecular dynamics: Density functional theory plus dispersion modelling of thermal motion in benzophenone," J. Appl. Crystallogr. 46(3), 656-662 (2013).

${ }^{13}$ A. Stone and S. Price, "Some new ideas in the theory of intermolecular forces: Anisotropic atom-atom potentials," J. Phys. Chem 92(12), 3325-3335 (1988).

${ }^{14}$ C. Andreani, R. Senesi, M. Krzystyniak, G. Romanelli, and F. Fernandez-Alonso, “Atomic quantum dynamics in materials research," Exp. Methods Phys. Sci. 49, 403-457 (2017)

${ }^{15}$ A. Parmentier, J. Shephard, G. Romanelli, R. Senesi, C. Salzmann, and C. Andreani, "Evolution of hydrogen dynamics in amorphous ice with density," J. Phys. Chem. Lett. 6(11), 2038-2042 (2015). 
${ }^{16}$ A. Parmentier, C. Andreani, G. Romanelli, J. J. Shephard, C. G. Salzmann, and R. Senesi, "Hydrogen mean force and anharmonicity in polycrystalline and amorphous ice," Front. Phys. 13(1), 136101 (2018).

${ }^{17} \mathrm{~B}$. Cheng, J. Behler, and M. Ceriotti, "Nuclear quantum effects in water at the triple point: Using theory as a link between experiments," J. Phys. Chem. Lett. 7(12), 2210-2215 (2016)

${ }^{18}$ D. Flammini, A. Pietropaolo, R. Senesi, C. Andreani, F. McBride, A. Hodgson, M. Adams, L. Lin, and R. Car, "Spherical momentum distribution of the protons in hexagonal ice from modeling of inelastic neutron scattering data," J. Chem. Phys. 136(2), 024504 (2012)

${ }^{19}$ G. Romanelli, M. Ceriotti, D. E. Manolopoulos, C. Pantalei, R. Senesi, and C. Andreani, "Direct measurement of competing quantum effects on the kinetic energy of heavy water upon melting," J. Phys. Chem. Lett. 4(19), 3251-3256 (2013).

${ }^{20}$ See www.isis.stfc.ac.uk for ISIS facility website.

${ }^{21} \mathrm{~V}$. Sears, "Scaling and final-state interactions in deep-inelastic neutron scattering," Phys. Rev. B 30(1), 44 (1984).

${ }^{22}$ J. Mayers and G. Reiter, "The VESUVIO electron volt neutron spectrometer," Meas. Sci. Technol. 23(4), 045902 (2012)

${ }^{23}$ G. Romanelli, M. Krzystyniak, R. Senesi, D. Raspino, J. Boxall, D. Pooley, S. Moorby, E. Schooneveld, N. Rhodes, C. Andreani et al., "Characterisation of the incident beam and current diffraction capabilities on the VESUVIO spectrometer," Meas. Sci. Technol. 28(9), 095501 (2017).

${ }^{24}$ A. Pietropaolo and R. Senesi, "Electron volt neutron spectrometers," Phys. Rep, 508(3), 45-90 (2011).

${ }^{25}$ E. Schooneveld, J. Mayers, N. Rhodes, A. Pietropaolo, C. Andreani, R. Senesi, G. Gorini, E. Perelli-Cippo, and M. Tardocchi, "Foil cycling technique for the VESUVIO spectrometer operating in the resonance detector configuration," Rev. Sci. Instrum. 77(9), 095103 (2006).

${ }^{26}$ D. Onorati, C. Andreani, L. Arcidiacono, F. Fernandez-Alonso, G. Festa, M. Krzystyniak, G. Romanelli, P. Ulpiani, and R. Senesi, "Gamma background characterization on VESUVIO: Before and after the moderator upgrade," J. Phys.: Conf. Ser. 1055(1), 012009 (2018).

${ }^{27}$ M. Tardocchi, A. Pietropaolo, C. Andreani, A. Bracco, A. D’Angelo, G. Gorini, S. Imberti, R. Senesi, N. J. Rhodes, and E. M. Schooneveld, "Cadmium-ZincTelluride photon detector for epithermal neutron spectroscopy-Pulse height response characterisation," Nucl. Instrum. Methods Phys. Res., Sect. A 526, 477-492 (2004)

${ }^{28}$ C. Andreani, A. Pietropaolo, R. Senesi, G. Gorini, M. Tardocchi, A. Bracco, N. J. Rhodes, and E. M. Schooneveld, "Electron-volt spectroscopy at a pulsed neutron source using a resonance detector technique," Nucl. Instrum. Methods Phys. Res., Sect. A 481, 509-520 (2002).

${ }^{29}$ M. Rebai, L. Giacomelli, C. Andreani, A. Fazzi, C. D. Frost, E. Perelli Cippo, A. Pietropaolo, N. J. Rhodes, M. Tardocchi, E. M. Schooneveld, and G. Gorini, "Diamond detectors for fast neutron measurements at pulsed spallation sources," J. Instrum. 7(05), C05015 (2012).

${ }^{30}$ A. Pietropaolo, C. Andreani, M. Rebai, L. Giacomelli, G. Gorini, E. Perelli Cippo, M. Tardocchi, A. Fazzi, G. Verona Rinati, C. Verona, M. Marinelli, E. Milani, C. D. Frost, and E. M. Schooneveld, "Fission diamond detectors for fast-neutron ToF spectroscopy," Europhys. Lett. 94(6), 62001 (2011).

${ }^{31}$ A. Pietropaolo, M. Tardocchi, E. M. Schooneveld, and R. Senesi, "Characterization of the background in epithermal neutron scattering measurements at pulsed neutron sources," Nucl. Instrum. Methods Phys. Res., Sect. A 568, 826-838 (2006).

${ }^{32}$ D. Onorati, "Optimization of gamma spectroscopy for energy analysis on $\mathrm{eV}$ neutron spectrometers," M.Sc. thesis, University of Rome Tor Vergata, 2017, http://purl.org/net/epubs/work/33481015.
${ }^{33}$ M. Tardocchi, G. Gorini, A. Pietropaolo, C. Andreani, R. Senesi, N. Rhodes, and E. M. Schooneveld, "YAP scintillators for resonant detection of epithermal neutrons at pulsed neutron sources," Rev. Sci. Instrum. 75, 4880 (2004).

${ }^{34}$ D. Anderson, T. Belgya, R. Firestone, Z. Kasztovsky, R. Lindstom, G. Molnár, Z. Révay, and C. Yonezawa, Handbook of Prompt Gamma Activation Analysis (Springer, USA, 2004).

${ }^{35}$ P. Ulpiani, G. Romanelli, L. Arcidiacono, D. Onorati, G. Festa, M. Krzystyniak, E. Schooneveld, F. Fernandez-Alonso, C. Andreani, and R. Senesi, "Enhancement of counting statistics and noise reduction in the forward-scattering detectors on the VESUVIO spectrometer," J. Phys.: Conf. Ser. 1055(1), 012008 (2018).

${ }^{36}$ See https://www.goodfellow.com/ for GoodFellow Website.

${ }^{37}$ E. Schooneveld, A. Pietropaolo, C. Andreani, E. P. Cippo, N. Rhodes, R. Senesi, M. Tardocchi, and G. Gorini, "Radiative neutron capture as a counting technique at pulsed spallation neutron sources: A review of current progress," Rep. Prog. Phys. 79(9), 094301 (2016).

${ }^{38}$ A. Miceli, G. Festa, R. Senesi, E. P. Cippo, L. Giacomelli, M. Tardocchi, A. Scherillo, E. Schooneveld, C. Frost, G. Gorini, and C. Andreani, "Measurements of gamma-ray background spectra at spallation neutron source beamlines," J. Anal. At. Spectrom. 29, 1897-1903 (2014).

${ }^{39}$ A. Pietropaolo, E. P. Cippo, G. Gorini, M. Tardocchi, E. Schooneveld, C. Andreani, and R. Senesi, " $\gamma$-ray background sources in the VESUVIO spectrometer at ISIS spallation neutron source," Nucl. Instrum. Methods Phys. Res., Sect. A 608(1), 121-124 (2009).

${ }^{40}$ J. Mayers, J. Tomkinson, T. Abdul-Redah, W. Stirling, C. Andreani, R. Senesi, M. Nardone, D. Colognesi, and E. Degiorgi, "VESUVIO-The double difference inverse geometry spectrometer at ISIS," Physica B 350(1), E659-E662 (2004).

${ }^{41} \mathrm{R}$. Senesi et al., "Time of flight-resolved gamma background measurements on VESUVIO," STFC ISIS Neutron and Muon Source (2017).

${ }^{42}$ C. Andreani, A. D’angelo, G. Gorini, S. Imberti, A. Pietropaolo, N. J. Rhodes, E. Schooneveld, R. Senesi, and M. Tardocchi, "CdZnTe $\gamma$ detector for deep inelastic neutron scattering on the VESUVIO spectrometer," Appl. Phys. A 78(6), 903-913 (2004).

${ }^{43}$ G. B. West, "Electron scattering from atoms, nuclei and nucleons," Phys. Rep. 18(5), 263-323 (1975).

${ }^{44}$ C. Andreani, G. Baciocco, R. Holt, and J. Mayers, "Resolution in deep inelastic neutron scattering using pulsed neutron sources," Nucl. Instrum. Methods Phys. Res., Sect. A 276, 297-305 (1989).

${ }^{45}$ S. Imberti, C. Andreani, V. Garbuio, G. Gorini, A. Pietropaolo, R. Senesi, and M. Tardocchi, "Resolution of the VESUVIO spectrometer for high-energy inelastic neutron scattering experiments," Nucl. Instrum. Methods Phys. Res., Sect. A 552(3), 463-476 (2005).

${ }^{46}$ J. Mayers, A. L. Fielding, and R. Senesi, "Multiple scattering in deep inelastic neutron scattering: Monte Carlo simulations and experiments at the ISIS eVS inverse geometry spectrometer," Nucl. Instrum. Methods Phys. Res., Sect. A 481(1-3), 454-463 (2002).

${ }^{47}$ G. Romanelli and M. Krzystyniak, "On the line-shape analysis of Compton profiles and its application to neutron scattering," Nucl. Instrum. Methods Phys. Res., Sect. A 819, 84-88 (2016)

${ }^{48}$ R. Senesi, G. Romanelli, M. Adams, and C. Andreani, “Temperature dependence of the zero point kinetic energy in ice and water above room temperature," Chem. Phys. 427, 111-116 (2013).

${ }^{49} \mathrm{See}$ https://root.cern.ch/ for ROOT data analysis framework Website.

${ }^{50}$ L. Lin, J. A. Morrone, R. Car, and M. Parrinello, "Displaced path integral formulation for the momentum distribution of quantum particles," Phys. Rev. Lett. 105(11), $110602(2010)$ 